\title{
Cyclin-dependent kinase 4 upregulation mediates acquired resistance of dabrafenib plus trametinib in BRAF V600E-mutated lung cancer
}

\author{
Noriko Hirai ${ }^{1} \wedge$, Yutaka Hatanaka ${ }^{2}$, Kanako C. Hatanaka ${ }^{2,3}$, Yuji Uno ${ }^{4}$, Shin-Ichi Chiba ${ }^{5}$, \\ Yasuhiro Umekage $^{1}$, Yoshinori Minami $^{1}$, Shunsuke Okumura ${ }^{1}$, Yoshinobu Ohsaki ${ }^{1,6}$, Takaaki Sasaki ${ }^{1 \wedge}$ \\ ${ }^{1}$ Respiratory Center, Asahikawa Medical University Hospital, Asahikawa, Japan; ${ }^{2}$ Research Division of Genome Companion Diagnostics, Hokkaido \\ University Hospital, Sapporo, Japan; ${ }^{3}$ Clinical Biobank, Clinical Research and Medical Innovation Center, Hokkaido University Hospital, Sapporo, \\ Japan; ${ }^{4}$ Department of Diagnostic Pathology, Asahikawa Medical University Hospital, Asahikawa, Japan; ${ }^{5}$ Center for Advanced Research and \\ Education, Asahikawa Medical University, Asahikawa, Japan; 'Yoshida Hospital, Asahikawa, Japan \\ Contributions: (I) Conception and design: N Hirai, T Sasaki; (II) Administrative support: N Hirai, T Sasaki; (III) Provision of study materials or patients: \\ N Hirai, T Sasaki; (IV) Collection and assembly of data: All authors; (V) Data analysis and interpretation: N Hirai, Y Hatanaka, KC Hatanaka, Y \\ Umekage, Y Minami, S Okumura, Y Ohsaki, T Sasaki; (VI) Manuscript writing: All authors; (VII) Final approval of manuscript: All authors. \\ Correspondence to: Takaaki Sasaki, MD, PhD. Respiratory Center, Asahikawa Medical University Hospital, Midorigaoka-Higashi-2-1-1-1, Asahikawa \\ 078-8510, Japan. Email: takaaki6@asahikawa-med.ac.jp.
}

\begin{abstract}
Background: Combination therapy with the B-Raf inhibitor, dabrafenib, and the MEK inhibitor, trametinib (DT) is commonly used to treat patients with B-Raf proto-oncogene, serine/threonine kinase V600E (BRAF V600E)-mutated non-small cell lung cancer (NSCLC). However, the mechanisms through which cancer develops DT resistance are unclear. Here, we investigated new mechanisms underlying acquired DT-resistant NSCLC with the BRAF V600E mutation.
\end{abstract}

Methods: We compared genomic signatures before and after DT treatment in patients with NSCLC.

Results: Two of four patients treated with DT developed carcinomatous pleuritis within 3 months. Target DNA sequencing and quantitative polymerase chain reaction (PCR) analyses revealed the increased expression level of cyclin-dependent kinase 4 (CDK4). We also found prominent protein expression of CDK4 after DT treatment. Induction of $C D K 4$ expression in a cell line derived from a patient with the $B R A F$ V600E mutation resulted in partial resistance to dabrafenib.

Conclusions: Our findings suggest a possible relationship between $C D K 4$ upregulation and acquired resistance to DT therapy.

Keywords: B-Raf proto-oncogene, serine/threonine kinase V600E (BRAF V600E); cell cycle; cyclin-dependent kinase 4 (CDK4); dabrafenib; trametinib

Submitted May 25, 2021. Accepted for publication Sep 12, 2021.

doi: $10.21037 /$ tlcr-21-415

View this article at: https://dx.doi.org/10.21037/tlcr-21-415

\section{Introduction}

Since the discovery of the oncogenic mutation in the B-Raf proto-oncogene, serine/threonine kinase V600E (BRAF V600E), combination therapy with the B-Raf inhibitor, dabrafenib, and the MEK inhibitor, trametinib (DT) has been used for treating BRAF V600E-mutated non-small cell lung cancer (NSCLC) (1). Although mutations in the mitogenactivated protein kinase (MAPK) or phosphatidylinositol

^ ORCID: Noriko Hirai, 0000-0002-5175-0800; Yutaka Hatanaka, 0000-0003-3128-1477; Takaaki Sasaki, 0000-0002-6505-8786. 
3-kinase (PI3K)/AKT pathways have been reported, the mechanisms of resistance to DT therapy are unclear (2-4).

Cyclin-dependent kinase $4(C D K 4)$ is involved in regulating cell proliferation during the G1 phase. CDK4 forms a complex with cyclin D, which is regulated by MAPK activity, thereby mediating retinoblastoma $(\mathrm{Rb})$ phosphorylation and resulting in cell cycle progression (5). CDK4/6 inhibitors have been used as anticancer agents in hormone receptorpositive breast cancer and KRAS-mutated NSCLC (6). Moreover, combined inhibition of $R A F$ and $C D K 4 / 6$ may have synergistic effects on tumors with $R A S$ or $R A F$ mutations (7). Additionally, CD4/6 inhibitors can overcome resistance to $B R A F$ inhibitors caused by cyclin $\mathrm{D} 1$ upregulation in $B R A F$ V600E-mutated melanoma (8). Thus, targeting CDK may overcome resistance to $R A S$ - and $R A F$-mutant cancers. $C D K 4$ may be implicated in the development of resistance in $B R A F$ V600E-mutant NSCLC; however, the involvement of CDK4 in acquired resistance is unclear.

Here, we established clinically resistant cell lines derived from pleural effusion samples of patients with NSCLC. We report two cases of clinically DT-resistant $B R A F$ V600Emutated NSCLC and compared the expression levels of cell cycle-related genes, including CDK4, in DT-naïve and DT-resistant cases. We present the following article in accordance with the STROBE reporting checklist (available at https://dx.doi.org/10.21037/tlcr-21-415).

\section{Methods}

The study was conducted in accordance with the Declaration of Helsinki (as revised in 2013). All study participants provided informed consent and this study was approved by the Institutional Review Board of Asahikawa Medical University (No.: 18185/19081). Written consent was obtained from all patients whose samples were analyzed, including pleural effusions.

\section{Patients and treatments}

Four patients were treated with DT, two of whom developed carcinomatous pleuritis after treatment. Pleural fluid from these two patients (LC-1, LC-6) was used for post-DT samples; lung tissue from corresponding patients collected before DT treatment was used for DT-naïve samples. The $B R A F$ V600E mutant (IG: pleural effusion; ON: transtracheal lymph node biopsy) and the BRAF K601E mutant (MY: pleural effusion) were also subjected to the Oncomine Dx target test (Thermo Fisher Scientific, Waltham, MA, USA), and cancer cells derived from DT-naïve patients harboring these genetic mutations were used as a reference.

\section{Establishment of cell line and expression constructs}

Lymph node biopsies of patients with the $B R A F_{-} V 600 \mathrm{E}$ mutation $(\mathrm{ON})$ were performed, and the excess specimens were used. The isolated lung cancer cells were cultured in RPMI 1640 medium (GIBCO, Thermo Fisher Scientific, Waltham, MA, USA) supplemented with $10 \%$ FBS, 100 units $/ \mathrm{mL}$ penicillin, $100 \mathrm{mg} / \mathrm{mL}$ streptomycin and $1 \mathrm{mmol} / \mathrm{L}$ sodium pyruvate as the primary culture medium. After confirming that the tumor cells were proliferating, single cells were cultured in 96-well plates, and only the proliferating lung cancer cells were passaged as cell lines.

The CDK4 cDNA vector was a gift from Sander van den Heuvel (Addgene, plasmid \#1876, Watertown, MA, USA). The CDK4 cDNA vector or empty vector was transfected by electrophoresis (Neon system, Thermo Fisher Scientific, Waltham, MA, USA). Dissociated ON cells were resuspended in $8 \mu \mathrm{L}$ of Neon Resuspension Buffer R for every one million cells. For each electroporation, cells and $2 \mu \mathrm{L}$ of vector were aliquoted into a sterile microcentrifuge tube. A Neon Tip was inserted into the Neon Pipette and the cell-DNA mixture was aspirated into the tip avoiding air bubbles. The Neon Pipette was then inserted into the Neon Tube containing $3 \mathrm{~mL}$ of Neon Electrolytic Buffer $\mathrm{E}$ in the Neon Pipette Station. Cells were pulsed twice with a voltage of 1,400 and a width of 20 . After the pulse, cells were quickly transferred into a RPMI1640 medium without antibiotics.

\section{Cell proliferation and growth assays}

Dabrafenib and erlotinib were purchased from Selleckchem (Boston, MA, USA) and inhibition of growth was assessed by MTS assay according to previously established methods (9). All experimental points were set up in 6 to 12 wells and all experiments were repeated 2 times.

\section{Genetic analysis}

$B R A F$ mutations were identified by the droplet digital polymerase chain reaction (PCR) or OncomineDx Target Test (Thermo Fisher Scientific, Waltham, MA, USA) in 12 of 233 patients with NSCLC over a 5 -year period (2015-2020) in Asahikawa Medical University Hospital. DNA was extracted from bronchial lavage fluid (QIAamp DNA Mini Kit, QIAGEN, Hilden, Germany). Digital 
PCR was performed using a $B R A F \mathrm{~V} 600 \mathrm{E}$-specific probe (Taqman cfDNA assay BRAF V600E, A44177 Thermo Fisher Scientific, Waltham, MA, USA) according to the QuantStudio3D (Thermo Fisher Scientific, Waltham, MA, USA) protocol. Genomic DNA mutations and copy number variations (CNVs) were assessed by a comprehensive cancer panel (160 genes, GeneRead DNAseq Targeted HC panel v2; QIAGEN, Hilden, Germany) on an Illumina MiSeq platform (Appendix 1).

\section{$m R N A$ expression analysis}

mRNA was extracted from formalin-fixed paraffinembedded samples of LC-1 and LC-6 samples using RNeasy Mini Kit (QIAGEN, Hilden, Germany). mRNA expression was assessed by the real-time PCR using a TaqMan Array human anticancer drug panel (RAPRJ2Y, Thermo Fisher Scientific, Waltham, MA, USA).

\section{Antibodies and Western blotting}

Cell lysis, Western blotting and immunohistochemistry (IHC) was done as previously described $(9,10)$. The following antibodies were used for immunostaining and Western blotting: anti-CDK4 (D9G3E), anti-cyclin D1 (92G2) and anti- $\beta$-actin (13E5) (Cell Signaling Technology, Danvers, MA, USA). The receptor tyrosine kinase (RTK) array (Proteome Profiler Human Phospho-RTK Array Kit, R\&D Systems, Minneapolis, MN, USA) was purchased and used according to the manufacturer's recommended conditions as previously described (9). List of 49 RTKs measuring relative phosphorylation status on antibody arrays were shown in Appendix 2.

\section{Statistical analysis}

In all cell growth assays, the mean and standard deviation were calculated from 6 wells of sample and 12 wells of control. The statistical analysis was carried out with twosample unpaired Student $t$-test performed using R (version 3.4.1; The R Foundation, Vienna, Austria) and 95\% CI of the mean difference was plotted.

\section{Results}

\section{Case LC-1}

A 61-year-old man was diagnosed with lung adenocarcinoma and underwent surgical resection of the right middle lobe (pathological stage 1A). Four years later, the adenocarcinoma recurred in the right upper lobe and metastasized to multiple lymph nodes, bone and the adrenal glands. The patient received four cycles of cisplatin/pemetrexed/bevacizumab, followed by maintenance therapy with pemetrexed/ bevacizumab. After five cycles of maintenance therapy, new metastases were found in the cervical lymph nodes. Following a cycle of pembrolizumab, the patient developed fever and hypoxemia owing to drug-induced pneumonia. The drug was discontinued and the patient was treated with oral steroids for 3 months. The BRAF V600E mutation was detected in the surgical specimen (LC-1 pre); thus, DT was administered as a third-line therapy. DT caused a temporary reduction in tumor size; however, the disease progressed to brain metastasis, meningitis and pleural effusion (LC-1 post) within 3 months. Whole-brain irradiation was performed, but the patient died 1 month after discontinuing DT.

\section{Case LC-6}

A 62 -year-old man was diagnosed with lung adenocarcinoma by transbronchial lymph node biopsy with bronchoscopy. Because the patient had clinical stage 3B disease, four cycles of cisplatin/pemetrexed/bevacizumab were administered, followed by maintenance therapy with pemetrexed/bevacizumab. After seven cycles of maintenance therapy, the patient was switched to nivolumab as secondline therapy owing to right lung progression. After 19 cycles of nivolumab, the patient was switched to docetaxel/ ramucirumab owing to right lung progression and lymph node metastasis. After 10 cycles of docetaxel/ramucirumab, the disease progressed with carcinomatous lymphangitis and bone metastasis. BRAF V600E was detected in a re-biopsy specimen of the mediastinal lymph nodes (LC-6 pre); thus, DT was started as a fourth-line therapy. After 11 months of DT, the disease progressed with pleural effusion (LC-6 post). The patient died 4 months after discontinuing DT.

\section{Genomic analysis and protein expression}

Next-generation sequencing was performed for LC-1 and LC-6 pre/post to evaluate acquired DT resistance (Figure 1, Table 1, Table S1). In LC-1, BRAF V600E was identified before and after DT treatment. The copy number variation (CNV) analysis showed that CDK4 on chromosome (chr) 12 was amplified after DT treatment. In LC-6, BRAF V600E was confirmed by SNV analysis before and after treatment, 

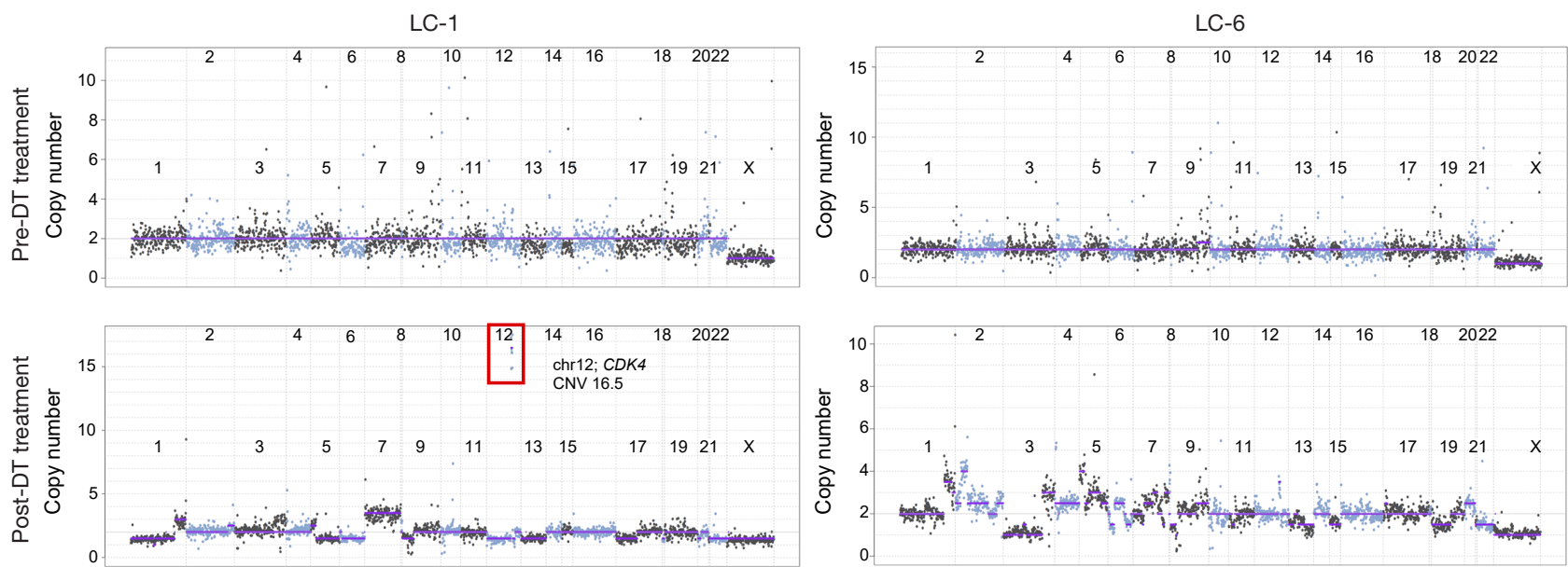

Figure 1 Changes in DNA copy number before and after treatment with DT in patients with BRAF V600E-mutated lung adenocarcinoma. Left panel: focal amplification $(\mathrm{CNV}=16.5)$ of the gene locus encoding $C D K 4$ at chr12 in specimens collected after the acquisition of resistance to DT in patient LC-1. Right panel: four copies of the gene on chrs 2 and 5, and copy number loss on chr 3 were observed in specimens collected after the acquisition of resistance in patient LC-6. DT, dabrafenib and trametinib; chr, chromosome; CDK4, cyclindependent kinase 4; BRAF V600E, B-Raf proto-oncogene, serine/threonine kinase V600E.

Table $1 \mathrm{CNV}$ in patients LC-1 and LC-6

\begin{tabular}{|c|c|c|c|c|c|c|}
\hline Cases & Chr & Gene name & Variant type & CNV change & $\begin{array}{l}\text { Pre-DT treatment } \\
\text { (copy number) }\end{array}$ & $\begin{array}{l}\text { Post-DT treatment } \\
\text { (copy number) }\end{array}$ \\
\hline \multirow[t]{9}{*}{ LC-1 } & Chr12 & $C D K 4$ & CNV & Gain & 2 & 16.5 \\
\hline & & PMS2 & & & & \\
\hline & & RAC1 & & & & \\
\hline & & $I K Z F 1$ & & & & \\
\hline & & $M E T$ & & & & \\
\hline & & SMO & & & & \\
\hline & & $B R A F$ & & & & \\
\hline & & $E Z H 2$ & & & & \\
\hline & Chr1 & MUTYH & CNV & Loss & 2 & 1.0 \\
\hline \multirow{9}{*}{ LC-6 } & Chr1 & $D D R 2$ & CNV & Gain & 2 & 3.5 \\
\hline & & $C D C 73$ & & & & \\
\hline & Chr3 & FANCD2 & CNV & Loss & 2 & 1 \\
\hline & & $V H L$ & & & & \\
\hline & & $X P C$ & & & & \\
\hline & & MLH1 & & & & \\
\hline & & SETD2 & & & & \\
\hline & & $B A P 1$ & & & & \\
\hline & & PBRM1 & & & & \\
\hline
\end{tabular}

CNV, copy number variation; chr, chromosome; DT, dabrafenib and trametinib. 
A

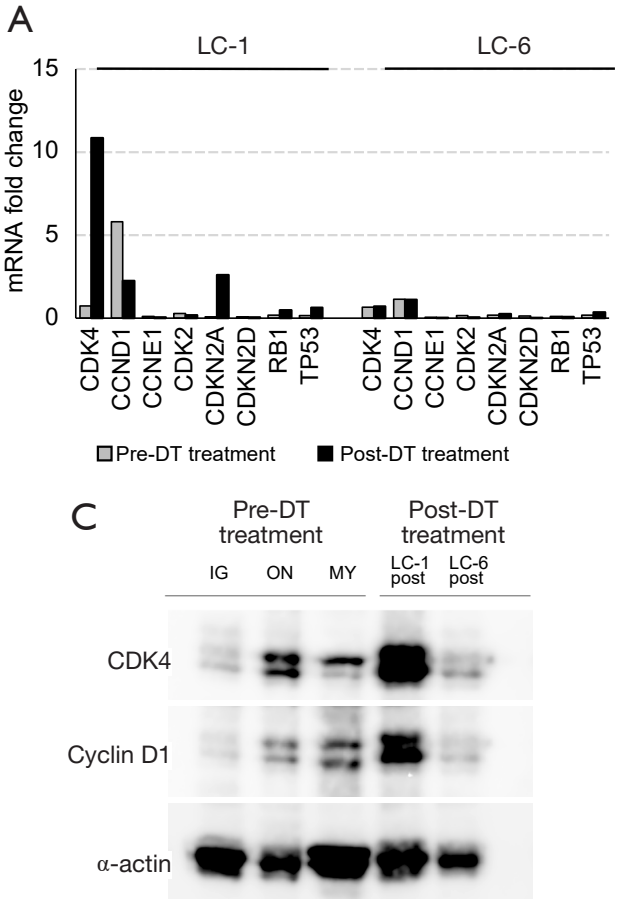

B
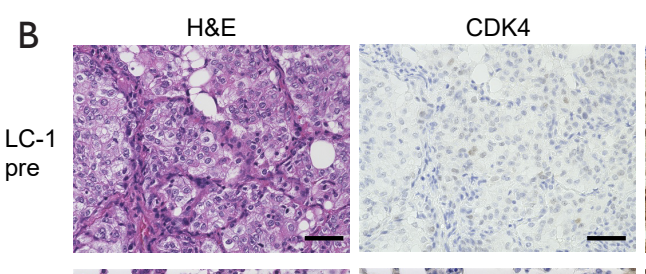

CyclinD1
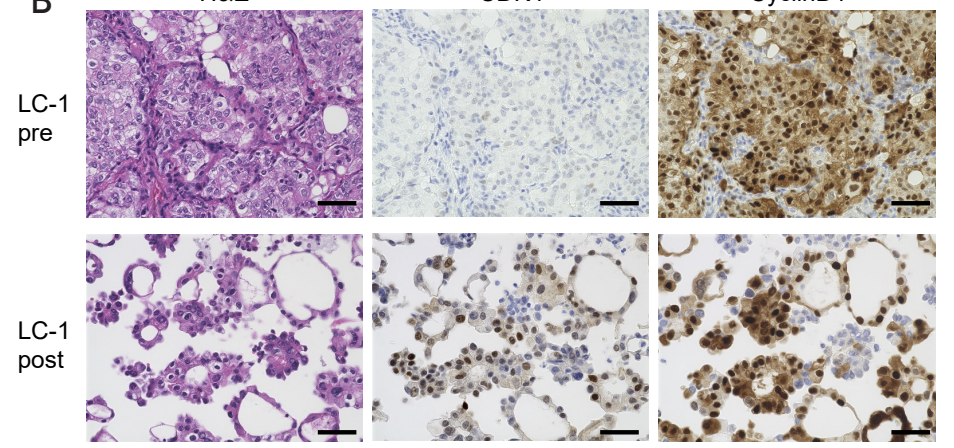

LC-6
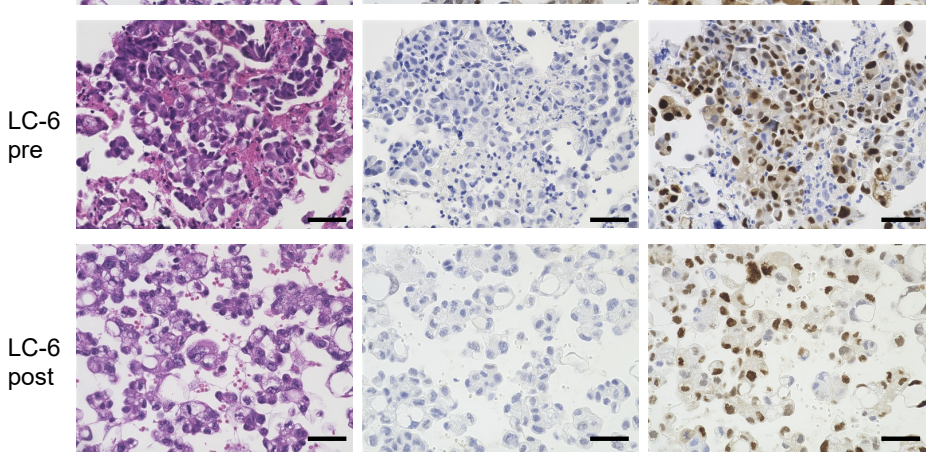

Figure 2 Investigation of mRNA expression and protein expression (using IHC and Western blotting) in samples from patients LC-1 and LC-6 before and after DT treatment. (A) Transcript levels of cell cycle-related genes determined using quantitative real-time PCR. (B) Microscopy-based images of H\&E, anti-CDK4 and anti-cyclin D1 staining before and after treatment with DT in samples from patients LC-1 and LC-6 (scale bar: $50 \mu \mathrm{m}$ ). (C) Protein expression in pleural effusion-derived cells from BRAF mutation-positive patients before and after DT treatment (IG: pleural effusion; ON: transtracheal lymph node biopsy; MY: pleural effusion). CDK4, cyclin-dependent kinase 4; $\mathrm{H} \& \mathrm{E}$, hematoxylin and eosin; DT, dabrafenib and trametinib; IHC, immunohistochemistry; PCR, polymerase chain reaction; BRAF, B-Raf proto-oncogene, serine/threonine kinase.

and missense S2264L and nonsense S2269* mutations in the AT-rich interaction domain $1 A$ (ARID1A) were detected on chr 1 in the post-DT sample. In both LC-1 and LC-6, other copy number aberrations were detected; however, there was no focal amplification directly associated with resistance.

Next, we performed quantitative PCR and IHC analyses on LC- 1 and LC-6 pre/post samples (Figure $2 A, 2 B$ ). In the LC-1 post sample, CDK4 mRNA was upregulated, as were the tumor suppressors $C D K N 2 A$ (encoding p16), $T P 53$, and $R b 1$. The $C D K 4$ protein was upregulated after DT therapy in LC-1, whereas CCND1 mRNA (encoding cyclin D1) was downregulated. However, cyclin D1 expression was observed before and after treatment, and cell cycle signatures were not markedly altered in LC-6 (Figure 2A,2B). Western blotting results (Figure 2C) showed that $C D K 4$ was expressed abundantly in LC-1, but only marginally in the other samples. Finally, we tested in vitro experiments to determine whether cells in the BRAF V600E cell line expressing the $C D K 4$ protein are resistant to $B R A F$ inhibitors. ON cells were transfected with a vector encoding CDK4 and treated with dabrafenib; at a concentration of $100 \mathrm{nM}$ dabrafenib, ON cells transfected with the CDK4 gene were more resistant than controls (Figure $3 A$ ). On the other hand, cells treated with erlotinib, an EGFR inhibitor, did not show any suppression of proliferation. The expression of $C D K 4$ protein was increased in $\mathrm{ON}$ cells transfected with a vector encoding CDK4 (Figure 3B). We further examined whether overexpression of $C D K 4$ causes a bypass pathway for $B R A F$ inhibitor resistance by increasing the phosphorylation of RTKs. The results showed that the phosphorylation of RTKs was not altered with or without forced expression of CDK4 protein (Figure 3C).

\section{Discussion}

Increased CDK4 DNA copy numbers followed by increased 

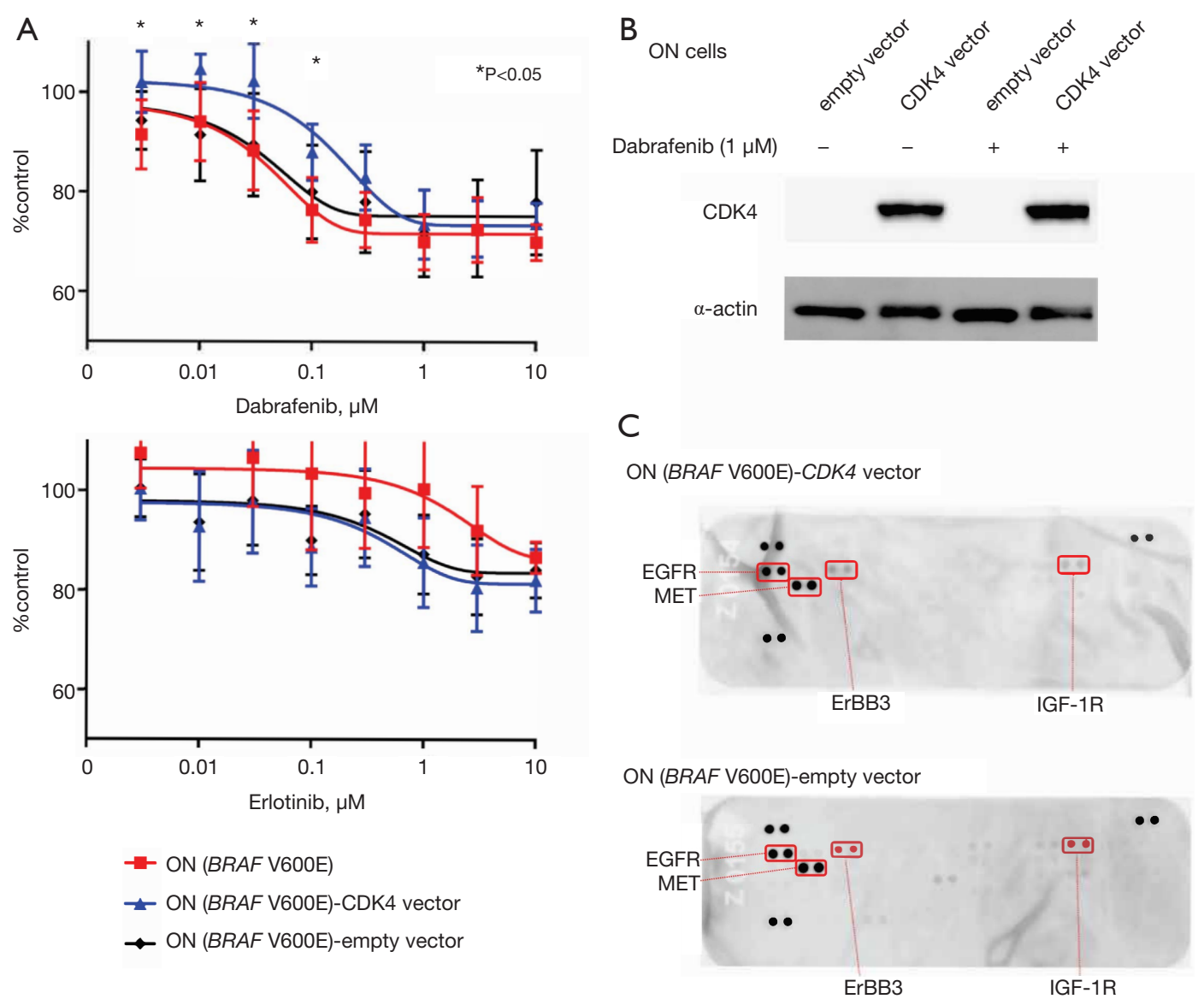

Figure 3 ON cell growth assays and RTK phosphorylation. (A) ON cells were treated with dabrafenib or erlotinib and cell proliferation was assessed by MTS assay (ON: transtracheal lymph node biopsy). CDK4 protein expressing cells were statistically resistance to dabrafenib at the concentration of 3-100 nM compared to control cells. (B) Protein expression of CDK4 was confirmed by Western blotting. (C) Changes in RTK phosphorylation status in ON cells expressing CDK4. Phosphorylation of EGFR, MET, ErBB3 and IGF-1R was also observed in control and $C D K 4$ expressing cells. We did not observe any RTKs altered by $C D K 4$ expression. * $\mathrm{P}<0.05$. CDK4, cyclin-dependent kinase 4; BRAF V600E, B-Raf proto-oncogene, serine/threonine kinase V600E; EGFR, epidermal growth factor receptor; MET, MET protooncogene, receptor tyrosine kinase; $E r B B 3$, erb-b2 receptor tyrosine kinase 3; $I G F-1 R$, insulin like growth factor 1 receptor; RTK, receptor tyrosine kinase.

mRNA and protein levels may be associated with the early acquisition of DT resistance in $B R A F \mathrm{~V} 600 \mathrm{E}$-mutated lung adenocarcinoma. Data from clinical trials show that the median duration of response to the DT combination DT is 9.8 months in previously treated patients with $B R A F$ V600E-mutated NSCLC, and the clinical course of LC-6 was similar. In contrast, LC-1 showed a short response period of about 3 months and rapidly worsened, exhibiting carcinomatous pleurisy, brain metastasis, and meningitis at relapse. Thus, there may be an association with an unknown resistance mechanism involving $C D K 4$.

Rudin et al. first reported resistance to dabrafenib in clinical specimens in $B R A F$ V600E-mutated adenocarcinoma in 2013 (4), KRAS G12D mutations and $C D K N 2 A$ nonsense mutations were the main causes of resistance. Previous case studies reporting acquired resistance to DT therapy in $B R A F$ V600E-mutated adenocarcinoma have detected KRAS G12V and NRAS Q61K mutations. Facchinetti et al. (3) performed a genetic analysis of eight $B R A F$ V600E-mutated lung cancers resistant to trametinib or dabrafenib and found mutations in MEK1, NRAS, KRAS and PTEN in four cases. Additionally, comparative genomic hybridization arrays were performed in the four cases in which no novel genetic mutations were identified by targeted next-generation 
sequencing, and deletions of $C D K N 2 A / B$ were found in two of these cases. Thus, the mechanisms of resistance to $B R A F$ or $B R A F / M E K$ inhibition include reactivation of the RAS/ MAPK pathway, supplementing signaling through other RAF family members, and activation of the PI3K/AKT pathway by PTEN deficiency. CDKN2 deletion or nonsense mutations are common in many cancers, although the significance of these mutations in the acquisition of DT resistance is unclear. Reactivation of the MAPK pathway and suppression of p16ink4 lead to positive regulation of the cell cycle; however, abnormalities in $R A S$ and $C D K N 2$ were not detected in our resistant cases.

Cyclin D-dependent kinases 4 and 6 are core proteins in the cell cycle machinery. In response to mitogenic signals, the cyclin D CDK4/CDK6/Cip/Kip complex is formed, resulting in the isolation of the $\mathrm{Cip} / \mathrm{Kip}$ proteins from cyclin E-CDK2. Cyclin D- and E-dependent kinases phosphorylate $\mathrm{Rb}$ protein, releasing $\mathrm{E} 2 \mathrm{~F}$, which is required for the transcription of genes necessary for $\mathrm{S}$ phase progression, including cyclin $\mathrm{E}$ itself, forming a positive feedback loop at the G1-S boundary. In malignant cells, alterations in the expression of CDK and its regulators, such as cyclin overexpression, regulate CDK activity and selectively promote proliferation (5). CDK4/6 inhibitors inhibit cell cycle progression and shows anti-tumor effects (6). Smalley et al. reported $C D K 4$ mutations or CCND1 amplification in BRAF V600E-mutated melanomas and evaluated their roles in resistance to $B R A F$ inhibition (11). They found that $C C N D 1$ amplification contributes to resistance to $B R A F$ inhibition, particularly in the presence of CDK4 mutations or overexpression. However, because LC-1 harbored only $C D K 4$ amplification, it is unclear whether the same resistance mechanism occurs in lung cancer.

The present in vitro study suggests that induction of CDK4 protein in BRAF V600E mutant cells results in dabrafenib resistance. The mechanism of resistance in lung cancer includes protein mutation due to the acquisition of gene mutation of therapeutic target and expression of bypass pathway mainly by RTKs. In these cells, the absence of secondary resistance mutations in $B R A F$ has been confirmed by the results of comprehensive genetic analysis. Therefore, we examined the phosphorylation of RTKs with an antibody array and did not find any specific changes. A weakness of this in vitro study is that ON cells are partially resistant to $B R A F$ inhibitors; the inhibition of cell proliferation by dabrafenib above $100 \mathrm{nM}$ was about $70 \%$, suggesting that this is a naturally acquired function during cell line establishment.

Taken together, these findings in LC-1 suggested that tumor cells originally harboring $B R A F \mathrm{~V} 600 \mathrm{E}$ mutations, and de novo or early acquired $C D K 4$ amplification, selectively survived under simultaneous $B R A F / M E K$ inhibition. We are currently investigating how $C D K 4$ contributes to the mechanism of resistance. Unfortunately, it was not possible to establish cell lines with pre-DT-treated samples. In IHC, cell blocks were used for post-DT samples because tissue biopsy was clinically difficult in these cases. Immunostaining of cell blocks may overestimate the intensity of staining. However, changes in $C D K 4$ staining of LC- 1 were evident.

In summary, CDK4 may be involved in the early acquisition of resistance and a new target for overcoming such resistance.

\section{Acknowledgments}

The authors would like to thank Editage for English language editing.

Funding: This work was supported by JSPS KAKENHI Grant Number JP18K08132. The funding source had no involvement in study design; in the collection, analysis, and interpretation of data; in the writing of the report; and in the decision to submit the article for publication.

\section{Footnote}

Reporting Checklist: The authors have completed the STROBE reporting checklist. Available at https://dx.doi. org/10.21037/tlcr-21-415

Data Sharing Statement: Available at https://dx.doi. org/10.21037/tlcr-21-415

Peer Review File: Available at https://dx.doi.org/10.21037/ tlcr-21-415

Conflicts of Interest: All authors have completed the ICMJE uniform disclosure form (available at https://dx.doi. org/10.21037/tlcr-21-415). Dr. Yutaka Hatanaka reports lecture fees from AstraZeneca and Novartis and research funds from Sysmex and Thermo Fisher Scientific. The authors have no conflicts of interest to declare.

Ethical Statement: The authors are accountable for all aspects of the work in ensuring that questions related to the accuracy or integrity of any part of the work are 
appropriately investigated and resolved. The study was conducted in accordance with the Declaration of Helsinki (as revised in 2013). All study participants provided informed consent and this study was approved by the Institutional Review Board of Asahikawa Medical University (No: 18185/19081). Written consent was obtained from all patients whose samples were analyzed, including pleural effusions.

Open Access Statement: This is an Open Access article distributed in accordance with the Creative Commons Attribution-NonCommercial-NoDerivs 4.0 International License (CC BY-NC-ND 4.0), which permits the noncommercial replication and distribution of the article with the strict proviso that no changes or edits are made and the original work is properly cited (including links to both the formal publication through the relevant DOI and the license). See: https://creativecommons.org/licenses/by-nc-nd/4.0/.

\section{References}

1. Planchard D, Besse B, Groen HJM, et al. Dabrafenib plus trametinib in patients with previously treated BRAF(V600E)-mutant metastatic non-small cell lung cancer: an open-label, multicentre phase 2 trial. Lancet Oncol 2016;17:984-93.

2. Abravanel DL, Nishino M, Sholl LM, et al. An Acquired NRAS Q61K Mutation in BRAF V600E-Mutant Lung Adenocarcinoma Resistant to Dabrafenib Plus Trametinib. J Thorac Oncol 2018;13:e131-3.

3. Facchinetti F, Lacroix L, Mezquita L, et al. Molecular mechanisms of resistance to BRAF and MEK inhibitors in BRAFV600E non-small cell lung cancer. Eur J Cancer

Cite this article as: Hirai N, Hatanaka Y, Hatanaka KC, Uno Y, Chiba SI, Umekage Y, Minami Y, Okumura S, Ohsaki Y, Sasaki T. Cyclin-dependent kinase 4 upregulation mediates acquired resistance of dabrafenib plus trametinib in $B R A F$ V600Emutated lung cancer. Transl Lung Cancer Res 2021;10(9):37373744. doi: $10.21037 /$ tlcr-21-415
2020;132:211-23.

4. Rudin CM, Hong K, Streit M. Molecular characterization of acquired resistance to the BRAF inhibitor dabrafenib in a patient with BRAF-mutant non-small-cell lung cancer. J Thorac Oncol 2013;8:e41-2.

5. Cole AM, Myant K, Reed KR, et al. Cyclin D2-cyclindependent kinase 4/6 is required for efficient proliferation and tumorigenesis following Apc loss. Cancer Res 2010;70:8149-58.

6. Patnaik A, Rosen LS, Tolaney SM, et al. Efficacy and Safety of Abemaciclib, an Inhibitor of CDK4 and CDK6, for Patients with Breast Cancer, Non-Small Cell Lung Cancer, and Other Solid Tumors. Cancer Discov 2016;6:740-53.

7. Chen SH, Gong X, Zhang Y, et al. RAF inhibitor LY3009120 sensitizes RAS or BRAF mutant cancer to CDK4/6 inhibition by abemaciclib via superior inhibition of phospho-RB and suppression of cyclin D1. Oncogene 2018;37:821-32.

8. Yadav V, Burke TF, Huber L, et al. The CDK4/6 inhibitor LY2835219 overcomes vemurafenib resistance resulting from MAPK reactivation and cyclin D1 upregulation. Mol Cancer Ther 2014;13:2253-63.

9. Sasaki T, Koivunen J, Ogino A, et al. A novel ALK secondary mutation and EGFR signaling cause resistance to ALK kinase inhibitors. Cancer Res 2011;71:6051-60.

10. Hirai N, Sasaki T, Okumura S, et al. Novel ALK-specific mRNA in situ hybridization assay for non-small-cell lung carcinoma. Transl Lung Cancer Res 2020;9:257-68.

11. Smalley KS, Lioni M, Dalla Palma M, et al. Increased cyclin D1 expression can mediate BRAF inhibitor resistance in BRAF V600E-mutated melanomas. Mol Cancer Ther 2008;7:2876-83. 


\section{Supplementary}

\section{Appendix 1-method}

List of 160 genes involved in cancer to be analyzed by GeneRead DNAseq Targeted HC panel v2

\begin{tabular}{llllllllll}
\hline ABL1 & AKT1 & AKT2 & ALK & AMER1 & APC & AR & ARID1A & ARID2 & ASXL1 \\
ATM & ATRX & BAP1 & BCL6 & BCOR & BRAF & BRCA1 & BRCA2 & BRIP1 & BTK \\
BUB1B & CARD11 & CBL & CBLB & CD79A & CD79B & CDC73 & CDH1 & CDK12 & CDK4 \\
CDKN2A & CHEK2 & CIC & CREBBP & CRLF2 & CSF1R & CTNNB1 & CYLD & DAXX & DDB2 \\
DDR2 & DICER1 & DNMT3A & ECT2L & EGFR & EP300 & EPCAM & ERBB2 & ERBB3 & ERBB4 \\
ERCC5 & ESR1 & EZH2 & FAM46C & FANCA & FANCD2 & FANCE & FAS & FBX011 & FBXW7 \\
FGFR2 & FGFR3 & FH & FLCN & FLT3 & FUBP1 & GATA1 & GATA2 & GATA3 & GNA11 \\
GNAQ & GNAS & GPC3 & GRIN2A & H3F3A & HIST1H3B & HNF1A & HRAS & HSPH1 & IDH1 \\
IDH2 & IKZF1 & IL6ST & IL7R & JAK1 & JAK2 & JAK3 & KDM6A & KDR & KIT \\
KLF6 & KMT2D & KRAS & MAP2K1 & MAP2K2 & MAP2K4 & MAP3K1 & MAP4K3 & MDM2 & MED12 \\
MEN1 & MET & MLH1 & MSH2 & MSH6 & MTOR & MUTYH & MYC & MYD88 & NF1 \\
NF2 & NFE2L2 & NFKBIA & NOTCH1 & NOTCH2 & NPM1 & NRAS & PALB2 & PAX5 & PBRM1 \\
PDGFRA & PHF6 & PIK3CA & PIK3R1 & PMS2 & PPP2R1A & PRDM1 & PRKAR1A & PTCH1 & PTEN \\
PTPN11 & RAC1 & RB1 & RET & ROS1 & SDHB & SETD2 & SF3B1 & SLC7A8 & SMAD4 \\
SMARCA4 & SMARCB1 & SMO & SPOP & SRC & STK11 & SUFU & TERT & TNFAIP3 & TNFRSF14 \\
TP53 & TSC1 & TSC2 & TSHR & U2AF1 & VHL & WT1 & XPC & ZNF2 & ZRSR2 \\
\hline
\end{tabular}

\section{Appendix 2-method}

List of 49 RTKs measuring relative phosphorylation status on antibody arrays

\begin{tabular}{lllllllllll}
\hline \multicolumn{2}{ll}{ ALK/CD246Axl } & DDR1 & DDR2 & Dtk & EGF R & EphA1 & EphA2 & EphA3 & EphA4 \\
EphA5 & EphA6 & EphA7 & EphA10 & EphB1 & EphB2 & EphB3 & EphB4 & EphB6 & ErbB2 \\
ErbB3 & ErbB4 & FGF R1 & FGF R2 $\alpha$ & FGF R3 & FGF R4 & Flt-3/Flk-2 & HGF R/c-MET & IGF-I R & Insulin R/CD220 \\
M-CSF R & Mer & MSP R/Ron & MuSK & PDGF R $\alpha$ & PDGF R $\beta$ & c-Ret & ROR1 & ROR2 & Ryk \\
SCF R/c-kitTie-1 & Tie-2 & TrkA & TrkB & TrkC & VEGF R1/Flt-1 & VEGF R2/KDR & VEGF R3/Flt-4
\end{tabular}

RTK, receptor tyrosine kinase. 
Table S1 Mutations and variants in patients LC-1 and LC-6

\begin{tabular}{lllllll}
\hline Cases & chr & Gene name & Variant type & $\begin{array}{l}\text { Amino acid } \\
\text { change }\end{array}$ & $\begin{array}{l}\text { Pre-DT treatment (mutant/wild-type } \\
\text { allele fraction) }\end{array}$ & $\begin{array}{l}\text { Post-DT treatment (mutant/wild-type } \\
\text { allele fraction) }\end{array}$ \\
\hline LC-1 & chr7 & BRAF & SNP & p.V600E & 0.177 & 0.570 \\
& chr17 & TP53 & SNP & p.V272M & 0.221 & 0.866 \\
& chr1 & H3F3A & SNP & p.R43W & 0.172 & 0.191 \\
& chr16 & GRIN2A & SNP & p.N1085Y & 0.174 & 0.590 \\
& chr12 & HNF1A & SNP & p.S487N & 0.341 & - \\
LC-6 & chr7 & BRAF & SNP & p.V600E & 0.060 & 0.776 \\
& chr1 & ARID1A & SNP & p.S2264L & - & 0.462 \\
\hline
\end{tabular}

chr, chromosome; DT, dabrafenib and trametinib; SNP, single nucleotide polymorphism. 